\title{
Satisfaction, Adverse Effects and Disclosure of Complementary and Alternative Medicine Use to Doctors in Urban Slum Areas of Ibadan, Nigeria: Variation by Ethnicity
}

\author{
Chinyerem C. Nwachukwu ${ }^{1 *}$, Amaechi C. Nwachukwu ${ }^{2}$, Akinola A. Fatiregun ${ }^{3}$ and Eme T. \\ Owoaje $^{4}$ \\ ${ }^{I}$ Department of Community Medicine, Chukwuemeka Odumegwu Ojukwu University, Awka, Nigeria \\ ${ }^{2}$ Department of Surgery, Chukwuemeka Odumegwu Ojukwu University, Awka, Nigeria \\ ${ }^{3}$ World Health Organization, Nigeria Country Office, Ondo State \\ ${ }^{4}$ Department of Community Medicine, University of Ibadan, Ibadan, Nigeria
}

*Corresponding Author: Chinyerem C. Nwachukwu, 1Department of Community Medicine, Chukwuemeka Odumegwu Ojukwu University, Awka, Nigeria, Email: chinyeremcynthia@yahoo.com

\begin{abstract}
Background: Sometimes people use medicines other than those prescribed by their doctors. Doctors are not always aware of their patients' use of these Complementary and Alternative Medicine (CAM), and why patients may choose not to disclose. This may have implications on drug-drug interaction.

Methods: A comparative cross-sectional study between two ethnic groups. The WHO modified cluster sampling technique was used to select 800 respondents, 400 from each of the study groups. Data was analysed using SPSS 17.0.

Results: Majority were satisfied with CAM use (Yoruba 86.3\%, Hausa 87.2\%), fewer were willing to use CAM in future (Yoruba 36.0\%, Hausa 31.7\%) and fewer still would recommend CAM to others (Yoruba 21.6\%, Hausa 21.0\%). Only a few respondents reported adverse effect from CAM use (Yoruba 8.7\%, Hausa $7.4 \%)$. The most reason given for non-disclosure of CAM use was the belief that doctors would not understand and would request the respondent to stop CAM use (Yoruba 9.3\%, Hausa 3.4\%). Also, doctor did not ask about CAM use (Yoruba 4.0\%, Hausa 6.5\%).
\end{abstract}

Conclusion: Further Research on various cam should be carried out to provide scientific information on efficacy, safety and adverse effects.

Keywords: Complementary, alternative, medicine, satisfaction, adverse effect, disclosure.

Abbreviation: Complementary and Alternative Medicine (CAM)

\section{INTRODUCTION}

Complementary and alternative medicine (CAM) is a group of diverse medical and health care systems, practices, and products that are not generally considered part of conventional medicine, whether they are indigenous or foreign to the culture [1]. For the purpose of this study, CAM has been classified into three: Unprocessed herbal preparations (unrefined herbal products and home preparations), Processed herbal preparations (pharmaceutically processed herbal products) and Other CAM (scarification, massage, python fat, local bone setting, ritual sacrifices, incantations, wearing of charms, rings, urine therapy).

CAM use has been reported to be beneficial by a number of studies [2-5]. More than $87 \%$ of American youth reported that they were 'helped a lot' by CAM [6]. Also, the majority of the elderly in an American study (80\%) reported that they had received substantial benefit from their use of CAM [7]. Many cancer patients in many European countries seemed to have benefited from using CAM, even though the benefits were not necessarily related to the 
initial reason for using CAM. Some $4.4 \%$ of patients, however, reported side-effects, mostly transient [8]. Most of the study respondents in Australia thought alternative medicines were safe but thought they were, or should be, subject to the same standards as prescribed medicines. Among respondents, 92.9\% wished product information to be of standard and content similar to those supplied with pharmaceuticals [9].

Ethno-medical research in Ibadan found that compounds of natural plants such as Calotropis $\mathrm{sp}$, Citrus sp and a few tropical plants have proved successful as a remedy for intra-uterine fibroids without surgery. The existing fibroid masses within the uterus gradually diminish in size, until they disappear completely. The regrowth of fibroid masses within the uterus is suppressed or inhibited [10]. A similar study showed that clinically confirmed cases of ovarian cysts were treated successfully without surgery in Ibadan using natural plant medicines made from the fibre of Cocos nucifera and other tropical plants [11]. Twenty five percent of children with chronic conditions who used CAM in Lagos could describe some specific benefits, while most of them (67.3\%) did not see any benefit from CAM use. More than $21 \%$ of these users reported various unwanted effects and $7.1 \%$ had adverse reactions [12].

Adverse effects have been reported in pregnant women and women of child-bearing age in London. The results of a study showed that prolonged use of echinacea may lead to toxicity of the liver and that prolonged use of ginseng could lead to unexplained vaginal bleeding and should be avoided by women taking oestrogens and warfarin and during the preconception and antenatal periods. Gingko biloba was found to crosses the placenta and was implicated in fetal anomalies. Raspberry leaf should only be taken in the third trimester in slowly increasing doses and should be avoided by women with a uterine scar, history of preterm labour and 'precious' pregnancy.[13] Some teenagers who were studied in Benin City revealed that they were introduced to alcohol drinking by their parents out of ignorance. The majority of the teenagers $(60 \%)$ reported that they started drinking alcohol concoction (mixture of alcohol and herbs) at home administered by their parents at a tender age [14].

Potential toxicity of herbal medicines due to their high levels of heavy metals [15], microbial contamination [16] and lack of safety warnings on their labels [17] are a source of concern. A study suggests that a Nigerian herbal supplement may have toxic effect on the spleen, pancreas, and heart of male albino rat [18]. On the contrary, T. Angelica herbal tonic, a herbal product used for indigestion and constipation and highly patronized in Nigeria, did not cause lethality and produced no signs of intoxication in mice. It exhibited a dose-dependent enhancement in the gastrointestinal tract motility, a modest fertility-promoting effect and showed lack of abortifacient and teratogenic properties [19].

Patients who were being managed for fracture in a secondary health facility in Ogbomoso were studied to compare the outcome of previous treatment. Over $50 \%$ of the patients who were previously treated by traditional bone setters had mal-union and a quarter had non-union. Only one of them (2.8\%) had no complaints and was satisfied with the outcome of treatment. Among those that were treated in the hospital from the onset, there were seven complications (14\%) and most of the complications involved the loss of joint motion [20]

Over 23\% of cancer patients in Enugu who used CAM were satisfied, but $68.3 \%$ were disappointed [21]. While $86.5 \%$ of these CAM users said that they will use orthodox medicine instead of CAM in the future, $9.6 \%$ said they will use the two together to help each other. Most of the users (79.8\%) will not repeat CAM or recommend its use [21].

Many respondents in a Lagos, Nigeria study, across the ethnic groups, had no idea of the constituents of the herbal remedies they use for treating their malaria episodes since they buy these from traditional herbalists. A majority of respondents claimed to use the same herbs for the treatment and prevention of malaria and experience great improvement after use [Hausas $(90 \%)$, Igbos $(83 \%)$, Yorubas $(77 \%)$ and the other ethnic groups (88\%)]. There is usually no specific dose or dose regimen, however, a high proportion in all the ethnic groups use herbal preparation thrice a day and a few of the respondents take unspecified measures at arbitrary intervals [22]. Nine percent of inpatients in a secondary health facility in Ibadan experienced adverse effects with the use of herbs, whereas $2 \%$ experienced adverse reactions on co-administration with prescribed drugs [23]. 
Only $53 \%$ of family practice patients in a study done in America had disclosed CAM use to their doctors [24]. The majority (72\%) of the respondents in America who used unconventional therapy did not inform their medical doctor that they had done so [25] Reasons given for non-disclosure included doctors not asking about CAM use, patients do not know that they should tell the doctor, beliefs that doctors do not need to know or would not understand, doctors' potentially negative responses to CAM and not enough time for discussion during the office visit $[1,12,21,26$ 28]. A United States based study showed that men were less likely than women to have discussed CAM with a doctor [1]. A study showed that in New York, America, disclosure of CAM use to medical providers was higher for provider-based CAM than self-care CAM. Disclosure of any CAM was associated with access to and quality of conventional care and higher among non-Latino whites relative to minorities. Having a regular doctor and quality patient-provider relationship removed racial/ethnic differences in CAM disclosure. Disclosure of any CAM use was lowest among Asian Americans (27\%) and highest among non-Latino whites $(41 \%)$. Relative to nonLatino whites, African-Americans, Latinos and Asian Americans were significantly less likely to disclose any CAM or self-care CAM. Compared to non-Latino whites, African Americans were more likely to discuss using provider-based CAM [29]. Another American study among those who use both alternative and orthodox medicine showed that only 33\% reported that they always tell their doctors about them [30].

An American based study among children in primary paediatric care practice showed that $81 \%$ of pediatric CAM users would have liked to discuss it with their pediatrician, but only $36 \%$ did so [31]. Many of the elderly in an American study (58\%) did not discuss the use of these therapies with their medical doctor [32]. A study on women with breast cancer in America showed that of those being treated by an alternative practitioner, $54 \%$ disclosed their CAM use to their physicians. Reasons for not disclosing CAM use included anticipating the physician's disinterest, negative response, or unwillingness or inability to contribute useful information; the perception that the CAM therapies used were irrelevant to the hospital treatment course; and the patients' views regarding the appropriate coordination of disparate healing strategies [33]. Many CAM users in Australia (57.2\%) did not tell their doctors about it [9].

\section{Materials ANd Methods}

\subsection{Study Area}

The study area was Ibadan, South-Western Nigeria. The indigenous tribe is Yoruba.

Two wards in Ibadan North Local Government Area were used - Sabo (predominantly Hausa residents) and Inalende (predominantly Yoruba residents) [34].

\subsection{Study Population}

The study population were adults aged 18 years and resident in selected study areas.

\subsection{Study Design}

The study was a comparative, cross-sectional study.

\subsection{Sample Size Determination}

The sample size was calculated using the formula for comparing two independent groups [35]:

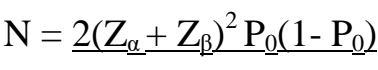

$$
\begin{aligned}
& d^{2}
\end{aligned}
$$

Where $\mathrm{N}=$ minimum sample size

$\mathrm{P}_{0}=$ mean prevalence of CAM use in the 2 comparison groups i.e. $\left(\mathrm{P}_{1+} \mathrm{P}_{2}\right) / 2$

$$
\frac{\mathrm{P} 1+\mathrm{P} 2}{2}=\frac{0.69+0.47}{2}=0.58
$$

$\mathrm{P}_{1}=0.69$. Previous estimate of CAM use among Yoruba population [36]

$\mathrm{P}_{2}=0.47$. Previous estimate of CAM use among Hausa population [36]

$\mathrm{d}=$ Difference between $\mathrm{P}_{1}$ and $\mathrm{P}_{2}=0.22$

$\mathrm{Z}_{\alpha}=$ Standard normal deviate corresponding to the probability $\alpha$, i.e. the probability of making a type 1 error at $5 \%=1.96$.

$\mathrm{Z}_{\beta}=$ the standard normal deviate at $80 \%$ statistical power, corresponding to the probability of making a type 2 error $=0.84$

$$
\begin{gathered}
\mathrm{N}=\frac{2(1.98+0.84)^{2} 0.58(1-0.58)}{0.22^{2}} \\
\mathrm{~N}=387
\end{gathered}
$$


A total of 400 respondents from each group were studied.

\subsection{Sampling Technique}

The sampling technique used was the World Health Organization (WHO) modified cluster sampling technique [37], adapted from the WHO 30 by 7 cluster sampling technique, developed by WHO in 1978 for estimating immunization coverage. A total of 400 questionnaires were administered in each ward. The ward settlements were considered as clusters and 50 questionnaires were administered in each of the 8 clusters (settlements) in Sabo and 67 in each of the first five clusters in Inalende and 65 in the sixth cluster.

\subsection{Research Instrument}

The research instrument was interviewer administered, semi-structured questionnaires.

\subsection{Data Collection}

Data was collected over a two-month period. Questionnaires were administered by trained research assistants who could speak the local language. The questionnaire was pretested in Ojoo in Akinyele LGA of Ibadan and appropriate amendments were made as necessary.

\subsection{Ethical consideration}

Approval for the study was obtained from the
Oyo State Ethical Review Committee. Informed consent was obtained from the respondents.

\subsection{Data Management}

Questionnaires were checked for errors and omissions at the end of each day. Data was analyzed using SPSS version 17.0. Frequencies, proportions, and means were compared between the two ethnic groups. Chi square test was used to test associations between categorical variables at 5\% level of significance.

\section{ReSUlts}

The sociodemographic characteristics showed some statistically significant differences between the two ethnic groups. Yorubas had an older population, more female respondents, had higher education and more professional and skilled workers. Hausas had a younger population, more male respondents and more partly skilled and unskilled workers. More Hausa respondents said that they will want to continue using CAM in future and to recommend to others. Most people did not disclose CAM use to their doctors. The most reason given was that they did not think that they should tell their doctor. A few adverse reactions from CAM use were reported. Also, Hausa respondents reported having more adverse reactions from unprocessed herbal preparations and it was statistically significant.

Table1. Respondents'socio-demographic characteristics

\begin{tabular}{|c|c|c|c|c|c|}
\hline $\begin{array}{l}\text { Socio-demographic } \\
\text { characteristics }\end{array}$ & $\begin{array}{l}\text { Yoruba } \\
\mathrm{N}=400 \mathrm{n}(\%)\end{array}$ & $\begin{array}{l}\text { Hausa } \\
\mathrm{N}=400 \text { n }(\%)\end{array}$ & $\begin{array}{l}\text { Total } \\
\mathbf{N}=800 \text { n }(\%)\end{array}$ & $\begin{array}{l}\text { Statistics } \\
\chi^{2}\end{array}$ & p-value \\
\hline $\begin{array}{l}\text { Age group (years) } \\
\quad \leq 24 \\
25-34 \\
35-44 \\
\quad \geq 45 \\
\text { Mean age }( \pm \text { SD) years } \\
\end{array}$ & $\begin{array}{l}40(10.0) \\
150(37.4) \\
75(18.8) \\
135(33.8) \\
40.1( \pm 15.8) \\
\end{array}$ & $\begin{array}{l}119(29.8) \\
137(34.2) \\
70(17.5) \\
74(18.5) \\
32.6( \pm 12.8)\end{array}$ & $\begin{array}{l}159(19.9) \\
287(35.9) \\
145(18.1) \\
209(26.1) \\
36.4(14.9) \\
\end{array}$ & $\begin{array}{l}57.817 \\
7.360 * * \\
\end{array}$ & $\begin{array}{l}<0.001 * \\
<0.001 *\end{array}$ \\
\hline $\begin{array}{ll}\text { Sex } & \\
& \text { Male } \\
& \text { Female } \\
\end{array}$ & $\begin{array}{l}165(41.2) \\
235(58.8) \\
\end{array}$ & $\begin{array}{l}209(52.2) \\
191(47.8) \\
\end{array}$ & $\begin{array}{l}374(46.8) \\
426(53.2) \\
\end{array}$ & 9.721 & $0.002 *$ \\
\hline $\begin{array}{c}\text { Level of education } \\
\text { No formal } \\
\text { Primary } \\
\text { Secondary } \\
\text { Tertiary } \\
\end{array}$ & $\begin{array}{l}59(14.8) \\
97(24.2) \\
210(52.5) \\
34(8.5) \\
\end{array}$ & $\begin{array}{l}95(23.8) \\
106(26.5) \\
187(46.7) \\
12(3.0) \\
\end{array}$ & $\begin{array}{l}154(19.2) \\
203(25.4) \\
397(49.6) \\
46(5.8)\end{array}$ & 1.081 & $<0.001 *$ \\
\hline $\begin{array}{l}\text { Marital status } \\
\text { Currently married } \\
\text { Not currently married }\end{array}$ & $\begin{array}{l}306(76.5) \\
94(23.5)\end{array}$ & $\begin{array}{l}243(60.8) \\
157(39.2)\end{array}$ & $\begin{array}{l}549(68.6) \\
251(31.4)\end{array}$ & 23.042 & $<0.001 *$ \\
\hline $\begin{array}{l}\text { Religion } \\
\text { Christianity } \\
\text { Islam } \\
\end{array}$ & $\begin{array}{l}191(47.8) \\
209(52.2) \\
\end{array}$ & $\begin{array}{l}6(1.6) \\
394(98.4) \\
\end{array}$ & $\begin{array}{l}197(24.6) \\
603(75.4) \\
\end{array}$ & 2.413 & $<0.001 *$ \\
\hline $\begin{array}{l}\text { Occupation } \\
\text { Professional \& Skilled } \\
\quad \text { Partly skilled } \\
\end{array}$ & $\begin{array}{l}142(35.5) 224 \\
(56.0)\end{array}$ & $\begin{array}{l}86(21.5) \\
254(63.5)\end{array}$ & $\begin{array}{l}228(28.4) \\
478(59.8)\end{array}$ & & \\
\hline
\end{tabular}


Satisfaction, Adverse Effects and Disclosure of Complementary and Alternative Medicine Use to Doctors in Urban Slum Areas of Ibadan, Nigeria: Variation by Ethnicity

\begin{tabular}{|c|c|c|c|c|c|}
\hline Unskilled & $34(8.5)$ & $60(15.0)$ & $94(11.8)$ & 22.829 & $<0.001 *$ \\
\hline $\begin{array}{r}\text { Monthly income } \\
<\$ 10,000 \\
\geq \$ 10,000 \\
(\$ 10,000 \approx \$ 26)\end{array}$ & $\begin{array}{l}245(61.2) \\
155(38.8)\end{array}$ & $\begin{array}{l}269(67.2) \\
131(32.8)\end{array}$ & $\begin{array}{l}514(64.2) \\
286(35.8)\end{array}$ & 3.135 & 0.077 \\
\hline
\end{tabular}

*Statistically significant

**t-test

Table2. Respondents' satisfaction with use of CAM

\begin{tabular}{|c|c|c|c|c|c|}
\hline CAM & $\begin{array}{l}\text { Yoruba } \\
\text { n }(\%)\end{array}$ & $\begin{array}{l}\text { Hausa } \\
\text { n }(\%)\end{array}$ & $\begin{array}{l}\text { Total } \\
\text { n }(\%)\end{array}$ & $\chi^{2}$ & p-value \\
\hline $\begin{array}{l}\text { Any CAM }(\mathbf{n}=\mathbf{6 5 2}) \\
\text { Yes } \\
\text { No }\end{array}$ & $\begin{array}{l}259(86.3) \\
41(13.7)\end{array}$ & $\begin{array}{l}307(87.2) \\
45(12.8)\end{array}$ & $\begin{array}{l}566(86.8) \\
86(13.2)\end{array}$ & 0.110 & 0.740 \\
\hline $\begin{array}{l}\text { Unprocessed } \quad \text { herbal } \\
\text { preparations }(\mathbf{n}=\mathbf{5 7 4}) \\
\text { Yes } \\
\text { No }\end{array}$ & $\begin{array}{l}238(90.0) \\
18(7.0)\end{array}$ & $\begin{array}{l}295(92.8) \\
23(7.2)\end{array}$ & $\begin{array}{l}533(92.9) \\
41(7.1)\end{array}$ & 0.009 & 0.926 \\
\hline $\begin{array}{l}\text { Processed } \\
\text { preparations }(\mathbf{n}=\mathbf{1 2 0}) \\
\text { Yes } \\
\text { No }\end{array}$ & $\begin{array}{l}31(77.5) \\
9(22.5)\end{array}$ & $\begin{array}{l}77(96.2) \\
3(3.8)\end{array}$ & $\begin{array}{l}108(90.0) \\
12(10.0)\end{array}$ & $\begin{array}{l}\text { Fisher's } \\
\text { exact }\end{array}$ & $0.002 *$ \\
\hline $\begin{array}{l}\text { Other CAM }(\mathbf{n}=\mathbf{3 1 7}) \\
\text { Yes } \\
\text { No }\end{array}$ & $\begin{array}{l}118(89.4) \\
14(10.6) \\
\end{array}$ & $\begin{array}{l}164(88.6) \\
21(11.4) \\
\end{array}$ & $\begin{array}{l}282(89.0) \\
35(11.0) \\
\end{array}$ & 0.044 & 0.835 \\
\hline
\end{tabular}

*Statistically significant

Table3. Respondents' willingness to continue using CAM in future

\begin{tabular}{|l|l|l|l|l|l|}
\hline Alternative medicine & $\begin{array}{l}\text { Yoruba } \\
\mathbf{n}(\boldsymbol{\%})\end{array}$ & $\begin{array}{l}\text { Hausa } \\
\mathbf{n}(\boldsymbol{\%})\end{array}$ & $\begin{array}{l}\text { Total } \\
\mathbf{n}(\boldsymbol{\%})\end{array}$ & $\chi^{\mathbf{2}}$ & p-value \\
\hline $\begin{array}{l}\text { Any CAM (n= 652) } \\
\text { Yes }\end{array}$ & $108(36.0)$ & $112(31.8)$ & $220(33.7)$ & & \\
No & $192(64.0)$ & $240(68.2)$ & $432(66.3)$ & 0.080 & 0.777 \\
\hline $\begin{array}{l}\text { Unprocessed herbal } \\
\text { preparations (n= 574) }\end{array}$ & & & & & \\
$\begin{array}{l}\text { Yes } \\
\text { No }\end{array}$ & $74(28.9)$ & $109(34.3)$ & $183(31.9)$ & & \\
\hline $\begin{array}{l}\text { Processed herbal } \\
\text { preparations (n= 120) }\end{array}$ & $182(71.1)$ & $209(65.7)$ & $391(68.1)$ & 1.884 & 0.170 \\
$\begin{array}{l}\text { Yes } \\
\text { No }\end{array}$ & $4(10.8)$ & $27(33.8)$ & $31(25.8)$ & & \\
\hline $\begin{array}{l}\text { Other CAM (n= 317) } \\
\text { Yes }\end{array}$ & $36(90.0)$ & $53(66.2)$ & $89(74.2)$ & 7.851 & $0.005^{*}$ \\
No & $82(62.1)$ & $139(75.1)$ & $221(69.7)$ & & \\
\hline
\end{tabular}

*Statistically significant

Table4. Respondents' willingness to recommend CAM use to other people

\begin{tabular}{|l|l|l|l|l|l|}
\hline CAM & $\begin{array}{l}\text { Yoruba } \\
\mathbf{n}(\boldsymbol{\%})\end{array}$ & $\begin{array}{l}\text { Hausa } \\
\mathbf{n}(\boldsymbol{\%})\end{array}$ & $\begin{array}{l}\text { Total } \\
\mathbf{n}(\boldsymbol{\%})\end{array}$ & $\chi^{2}$ & p-value \\
\hline $\begin{array}{l}\text { Any CAM (n= 652) } \\
\text { Yes }\end{array}$ & $\begin{array}{l}6(21.6) \\
\text { No }\end{array}$ & $\begin{array}{l}73(21.0) \\
274(79.0)\end{array}$ & $\begin{array}{l}139(21.3) \\
513(78.7)\end{array}$ & 0.035 & 0.851 \\
\hline $\begin{array}{l}\text { Unprocessed herbal } \\
\text { preparations (n= 574) }\end{array}$ & $62(23.3)$ & $52(16.9)$ & $114(19.9)$ & & \\
$\begin{array}{l}\text { Yes } \\
\text { No }\end{array}$ & $204(76.7)$ & $256(83.1)$ & $460(80.1)$ & 3.702 & 0.054 \\
\hline $\begin{array}{l}\text { Processed herbal } \\
\text { preparations (n= 120) }\end{array}$ & & & & & \\
\hline
\end{tabular}


Satisfaction, Adverse Effects and Disclosure of Complementary and Alternative Medicine Use to Doctors in Urban Slum Areas of Ibadan, Nigeria: Variation by Ethnicity

\begin{tabular}{|l|l|l|l|l|l|}
\hline Yes & $\begin{array}{l}\text { (10.0) } \\
\text { No }\end{array}$ & $\begin{array}{l}22(27.5) \\
58(72.5)\end{array}$ & $\begin{array}{l}26(21.7) \\
94(78.3)\end{array}$ & 4.812 & $0.028^{*}$ \\
\hline Other CAM (n= 317) & $17(12.9)$ & $60(32.4)$ & $77(24.3)$ & & \\
Yes & $115(87.1)$ & $125(67.6)$ & $240(75.7)$ & 16.016 & $<0.001 *$ \\
No & &
\end{tabular}

*Statistically significant

Table5. Disclosure of CAM use to doctors

\begin{tabular}{|c|c|c|c|c|c|}
\hline Variable & $\begin{array}{l}\text { Yoruba } \\
\text { n }(\%)\end{array}$ & $\begin{array}{l}\text { Hausa } \\
\text { n }(\%)\end{array}$ & $\begin{array}{l}\text { Total } \\
\text { n }(\%)\end{array}$ & $\chi^{2}$ & p-value \\
\hline Disclosed & $109(30.7)$ & $110(28.7)$ & $219(29.7)$ & & \\
\hline Not disclosed & $245(69.3)$ & $273(71.3)$ & $518(70.3)$ & 4.176 & 0.653 \\
\hline Total & $354(100.0)$ & $383(100.0)$ & $737(100.0)$ & & \\
\hline
\end{tabular}

Table6. Reasons for non-disclosure of CAM use to doctors

\begin{tabular}{|c|c|c|c|c|c|}
\hline $\begin{array}{l}\text { Reasons for non- } \\
\text { disclosure of CAM use }\end{array}$ & $\begin{array}{l}\text { Yoruba } \\
\text { n }(\%)\end{array}$ & $\begin{array}{l}\text { Hausa } \\
\text { n }(\%)\end{array}$ & $\begin{array}{l}\text { Total } \\
\text { n }(\%)\end{array}$ & $\begin{array}{l}\text { Statistics } \\
\chi 2\end{array}$ & p-value \\
\hline & $(n=245)$ & $(n=273)$ & $(n=518)$ & & \\
\hline $\begin{array}{l}\text { I did not think that I } \\
\text { should tell the doctor } \\
\text { Yes } \\
\text { No }\end{array}$ & $\begin{array}{l}193(78.8) \\
52(21.2)\end{array}$ & $\begin{array}{l}237(86.8) \\
36(13.2)\end{array}$ & $\begin{array}{l}430(83.0) \\
88(17.0)\end{array}$ & 5.915 & 0.015 \\
\hline $\begin{array}{l}\text { Doctors would not } \\
\text { understand and would ask } \\
\text { me to stop } \\
\text { Yes } \\
\text { No }\end{array}$ & $\begin{array}{l}23(9.3) \\
222(90.7)\end{array}$ & $\begin{array}{l}28(7.3) \\
245(92.7)\end{array}$ & $\begin{array}{l}51(6.9) \\
467(93.1) \\
\end{array}$ & 0.110 & 0.740 \\
\hline $\begin{array}{l}\text { Doctor did not ask about } \\
\text { alternative medicine use } \\
\text { Yes } \\
\text { No }\end{array}$ & $\begin{array}{l}14(4.0) \\
231(96.0)\end{array}$ & $\begin{array}{l}25(6.5) \\
248(93.5)\end{array}$ & $\begin{array}{l}39(5.3) \\
479(94.7)\end{array}$ & 2.199 & 0.138 \\
\hline $\begin{array}{l}\text { There wasn't enough time } \\
\text { during the consultation } \\
\text { with the doctor } \\
\text { Yes } \\
\text { No }\end{array}$ & $\begin{array}{l}11(3.1) \\
234(96.9)\end{array}$ & $\begin{array}{l}9(23.5) \\
266(76.5)\end{array}$ & $\begin{array}{l}20(2.7) \\
498(97.3)\end{array}$ & 0.519 & 0.471 \\
\hline $\begin{array}{l}\text { I have not been to a } \\
\text { hospital } \\
\text { Yes } \\
\text { No }\end{array}$ & $\begin{array}{l}4(1.1) \\
241(98.9)\end{array}$ & $\begin{array}{l}2(0.5) \\
273(99.5)\end{array}$ & $\begin{array}{l}6(0.8) \\
512(99.2)\end{array}$ & $\begin{array}{l}\text { Fisher's } \\
\text { exact }\end{array}$ & 0.580 \\
\hline
\end{tabular}

Table7. Adverse effects experienced by CAM users

\begin{tabular}{|c|c|c|c|c|c|}
\hline CAM & $\begin{array}{l}\text { Yoruba } \\
\text { n }(\%)\end{array}$ & $\begin{array}{l}\text { Hausa } \\
\text { n }(\%)\end{array}$ & $\begin{array}{l}\text { Total } \\
\text { n }(\%)\end{array}$ & $\chi^{2}$ & p-value \\
\hline $\begin{array}{l}\text { Any CAM }(\mathbf{n}=\mathbf{6 5 2}) \\
\text { Yes } \\
\text { No }\end{array}$ & $\begin{array}{l}26(8.7) \\
274(91.3)\end{array}$ & $\begin{array}{l}26(7.4) \\
326(92.6)\end{array}$ & $\begin{array}{l}52(8.0) \\
600(92.0) \\
\end{array}$ & 0.362 & 0.548 \\
\hline $\begin{array}{l}\text { Unprocessed } \quad \text { herbal } \\
\text { preparations }(\mathbf{n}=\mathbf{5 7 4}) \\
\text { Yes } \\
\text { No }\end{array}$ & $\begin{array}{l}10(3.9) \\
246(96.1)\end{array}$ & $\begin{array}{l}35(11.0) \\
283(89.0)\end{array}$ & $\begin{array}{l}45(7.8) \\
529(92.2)\end{array}$ & 9.895 & $0.002 *$ \\
\hline $\begin{array}{l}\text { Processed herbal } \\
\text { preparations }(\mathbf{n}=\mathbf{1 2 0}) \\
\text { Yes } \\
\text { No }\end{array}$ & $\begin{array}{l}2(5.3) \\
36(94.7)\end{array}$ & $\begin{array}{l}3(3.8) \\
77(36.2)\end{array}$ & $\begin{array}{l}5(4.2) \\
115(95.8)\end{array}$ & 0.104 & 0.747 \\
\hline $\begin{array}{l}\text { Other CAM }(\mathbf{n}=\mathbf{3 1 7}) \\
\text { Yes } \\
\text { No }\end{array}$ & $\begin{array}{l}4(3.0) \\
128(97.0)\end{array}$ & $\begin{array}{l}18(9.7) \\
167(90.3)\end{array}$ & $\begin{array}{l}22(6.9) \\
295(93.1)\end{array}$ & 5.353 & 0.021 \\
\hline
\end{tabular}




\section{DisCUSSION}

Majority (over four-fifth) of respondents in this study were satisfied with CAM use. However, only about one-fifth of respondents of cancer patients in Enugu were satisfied with CAM use while the rest were disappointed [21]. This is understandable because our study participants used CAM for the treatment of a variety of disease conditions as compared to cancer which has poor prognosis in our environment. Other studies among American youth [6] and American elders [7] reported substantial benefits from CAM use from over four-fifth of respondents, which is similar to the result of this study. The proportion of this study respondents who are willing to continue using CAM $33.7 \%$ and recommend them to other people $21.3 \%$, is similar to findings among respondents in Enugu where $20.2 \%$ of respondents were willing to continue use and to recommend CAM [21].

Very few respondents reported experiencing adverse reactions with the use of CAM in this study $(8.0 \%)$ which is similar to the $9 \%$ reported in Ibadan [38], 7.1\% in Lagos state and $4.4 \%$ in Australia [39].

No significant ethnic difference was found with regards to disclosure of CAM use to doctors though more Yoruba respondents $30.7 \%$ than Hausa respondents $28.7 \%$ had done so. This is similar to the findings of a study done among family practice patients in America where there was no association between race and disclosure [24]. However, this differed from the findings of a multi-ethnic study where disclosure was lowest among Asian Americans 27\% and highest among non-Latino whites $41 \%$. It was also found that African-Americans, Latinos and Asian-Americans were significantly less likely to disclose CAM use than non-Latino whites [30]. Generally the fewer respondents in this study disclosed CAM use when compared with CAM users in Australia 57.2\% [39], the elderly $58 \%$ [7], family practice patients in America [24] and breast cancer patients in America 54\% [40]. Similar reasons for non-disclosure was given in all studies, the most reason being that the doctor would not understand and would advise the respondent to discontinue.

\section{CONCLUSION}

Although, the majority of users expressed satisfaction with CAM use, few were willing to continue use or to recommend CAM to others. The proportions reporting adverse effects were few. Hausa respondents reported having more adverse reactions from unprocessed herbal preparations and it was statistically significant. Many users of CAM did not disclose use to their doctors and this was similar for both ethnic groups.

\section{REFERENCES}

[1] National Centre for Complementary and Alternative Medicine. The use of Complementary and Alternative Medicine in the United States 2007.

[2] Shenfield G, Lim E, Allen H. Survey of the use of complementary medicines and therapies in children with asthma. J Paediatr Child Health. 2002;38:252-7.

[3] Sibinga E, Shindell DL, Casella JP, Duggan AK, Wilson MH. Paediatric patients with sickle cell disease: use of complementary and alternative therapies. J Alternat Complement Med 2006.12:291-8.

[4] Martel D, Bussieres J, Theoret Y, Lebel D, Kish S, Moghrabi A, Laurier Cl. Use of alternative and complementary therapies in children with cancer. Paediatr Blood Cancer. 2005;44:660-8.

[5] Smith C, Eckert K. Prevalence of complementary and alternative medicine and use among children in South Australia. J Paediatr Child Health. 2006;42:538-43.

[6] Breuner C, Barry P, Kemper K. Alternative Medicine Use by Homeless Youth. Arch Pediatr Adolesc Med. 1998;152:1071-5.

[7] Astin J, Pelletier K, Marie A, Haskell W. Complementary and alternative medicine use among elderly persons: one-year analysis of a Blue Shield Medicare supplement. J Gerontol A Biol Sci Med Sci. 2000;55(1):M4-9.

[8] Molassiotis A, Fernadez-Ortega P, Pud D, Ozden G, Scott JA, Panteli V, et al. Use of complementary and alternative medicine in cancer patients: a European survey. Ann Oncol 2005;16(4):655-63.

[9] MacLennan A, Wilson D, Taylor A. The Escalating Cost and Prevalence of Alternative Medicine Preventive Medicine. 2002; 35(2) : 166-73.

[10] Olapade EO, Olapade CAO, Olapade CO, Olapade JB. Recent Successes in the Use of Natural Herbal Remedies for the Treatment of Intra-uterine Fibroids Without Surgery. HortScience. 2004;39:860-1.

[11] Olapade EO, Olapade EOJ, Olapade CAO, Olapade CO, Olapade JB. Natural Plants Treatment for Ovarian Cysts in Women Without Surgery. American Society for Horticultural Science. 2004;39:860. 
[12] Kazeem AO, Idowu OS, Olisamedua FN, Ayo S. Use of complementary and alternative medicine for children with chronic conditions in Lagos, Nigeria2008: Available from: http// www.biomedcentral.com/1472-6882/8/66.

[13] Tiran D. The use of herbs by pregnant and child bearing women: a risk-benefit assessment. Complementary Therapies in Nursing \& Midwifery. 2003;9:176-81.

[14] Oshodin OG. Parental influences upon alcohol use by teenagers in Benin City, Nigeria. Perspectives in Public Health. 1984;104 (3): 106-7.

[15] Obi E, Akunyili DN, Ekpo B, Orisakwe OE. Heavy metal hazards of Nigerian herbal remedies. Sci Total Environ. 2006;369:35-41.

[16] Efuntoye M. Mycotoxins of fungal strains from stored herbal plants and mycotoxin contents of Nigerian crude herb drugs. Mycopathologica. 1999;147:43-8.

[17] Obi E, Agbasi PU, Ezejiofor NA, Maduagwuna C, Orisakwe OE. Safety warnings and first aid instructions on Nigerian traditional herbal remedies: are they adequate. World J Med Sci. 2006;1:108-11.

[18] Ezejiofor NA, Maduagwunan, Onyiaorah VI, Hussaini DC, Orisakwe OE. Multiple organ toxicity of a Nigerian herbal supplement (U \& D Sweet bitter) in male albino rats. Pak J Pharm Sci. 2008 Oct;21(4):426-9.

[19] Esimone CO, Akah PA, Nworu CS. Efficacy and Safety Assessment of T. Angelica Herbal Tonic ${ }^{\circ}$, a Phytomedicinal Product Popularly Used in Nigeria. Evidence based Complementary and Alternative Medicine. 2009 September 13.

[20] OlaOlorun DA, Oladiran IO, Adeniran A. Complications of fracture treatment by traditional bonesetters in southwest Nigeria. Family Practice. 2001;18:635-7.

[21] Ezeome E, Anarado AN. Use of complementary and alternative medicine by cancer patients at the University of Nigeria Teaching Hospital, Enugu, Nigeria. BMC Complement Altern Med. 2007 Sept;12(7):28.

[22] Idowu E, Mafe MA, Otubanjo OA, Adeneye AK. Herbal remedy in the treatment of malaria: cross sectional survey of residents of Lagos State, Nigeria. Afr J Med Med Sci. 2006 June;35(2):149-53.

[23] Fakeye T, Tijani A, Adebisi O. A survey of the use of herbs among patients attending secondary-level health care facilities in southwestern Nigeria. J Herb Pharmacother. 2007;7(3-4):213-27.

[24] Elder NC, Gillcrist A, Minz R. Use of Alternative Health Care by Family Practice Patients. Arch Fam Med. 1997;6(2):181-4.
[25] Eisenberg DM, Kessler RC, Foster C, Frances E. Norlock FE, Calkins DR, Delbanco TL. Unconventional Medicine in the United States - Prevalence, Costs, and Patterns of Use. N Engl J Med. 1993;328:246-52.

[26] Eisenberg DM, Kessler RC, Rompay MIV, et al. Perceptions about complementary therapies relative to conventional therapies among adults who use both: results from a national survey. Ann Intern Med. 2001;135:344-51.

[27] Robinson A, McGrail M. Disclosure of CAM use to medical practitioners: a review of qualitative and quantitative studies. Complement Ther Med. 2004;12:90-8.

[28] Tasaki K, Maskarinec G, Shumay D, et al. Communication between physicians and cancer patients about complementary and alternative medicine: exploring patients' perspectives. Psychooncology. 2002;11:212-20.

[29] Chao M, Wade C, Kronenberg F. Disclosure of Complementary and Alternative Medicine to Conventional Medical Providers: Variation by Race/Ethnicity and Type of CAM. J Natl Med Assoc. 2008;100(11):1341-9.

[30] Archer EL, Boyle DK. Herb and Supplement Use Among the Retail Population of an Independent, Urban Herb Store. J Holist Nurs. 2008;26(1):27-35.

[31] Ottolini MCA, Hamburger EKA, Loprieato JOA, Coleman RHA, Hari C.Â Sachs HCA, Madden RA, et al. Complementary and Alternative Medicine Use Among Children in the Washington, DC Area. Academic Pediatrics. 2001;1(2):122-5.

[32] Astin J, Pelletier K, Marie A, Haskell W. Complementary and alternative medicine use among elderly persons: one-year analysis of a Blue Shield Medicare supplement. J Gerontol A Biol Sci Med Sci. 2000;55(1):M4-9.

[33] Adler S, Fosket J. Disclosing complementary and alternative medicine use in the medical encounter: a qualitative study in women with breast cancer. J Fam Pract. 1999;48(6):453-8.

[34] National Bureau of Statistics. Federal Republic of Nigeria 2006 population census.

[35] Kelsey JL, editor. Methods in observational epidemiology. USA: Oxford University Press; 1996.

[36] Idowu E, Mafe MA, Otubanjo OA, Adeneye AK. Herbal remedy in the treatment of malaria: cross sectional survey of residents of Lagos State, Nigeria. Afr J Med Med Sci. 2006 June; 35(2):149-53.

[37] World Health Organization. The EPI coverage survey1991.

[38] Fakeye T, Tijani A, Adebisi O. A survey of the use of herbs among patients attending secondary-level health care facilities in 
Satisfaction, Adverse Effects and Disclosure of Complementary and Alternative Medicine Use to Doctors in Urban Slum Areas of Ibadan, Nigeria: Variation by Ethnicity

southwestern Nigeria. J Herb Pharmacother. 2007;7(3-4):213-27.

[39] MacLennan A, Wilson D, Taylo A. The Escalating Cost and Prevalence of Alternative Medicine. Preventive Medicine. 2002; 35(2) : 166-73.
[40] Adler S, Fosket J. Disclosing complementary and alternative medicine use in the medical encounter: a qualitative study in women with breast cancer. J Fam Pract. 1998;48(6):453-8.

Citation: Chinyerem C. Nwachukwu, et.al, Satisfaction, Adverse Effects and Disclosure of Complementary and Alternative Medicine Use to Doctors in Urban Slum Areas of Ibadan, Nigeria: Variation by Ethnicity. ARC Journal of Public Health and Community Medicine. 2021; 6(2):08-16. Doi:doi.org/10.20431/2456-0596.060 1002.

Copyright: () 2021 Authors. This is an open-access article distributed under the terms of the Creative Commons Attribution License, which permits unrestricted use, distribution, and reproduction in any medium, provided the original author and source are credited. 